ISSN 1561-8358 (Print)

ISSN 2524-244X (Online)

УДК 621.785 .532

https://doi.org/10.29235/1561-8358-2018-63-3-342-350

Поступила в редакцию 07.06.2018

Received 07.06.2018

\author{
М. Н. Босяков ${ }^{1}$, А. А. Козлов ${ }^{2}$ \\ ${ }^{I}$ Физико-технический институт Национальной академии наук Беларуси, Минск, Беларусь \\ ${ }^{2}$ Министерство промышленности Республики Беларусь, Минск, Беларусь

\section{ЭНЕРГЕТИЧЕСКИЕ И ГАЗОДИНАМИЧЕСКИЕ ХАРАКТЕРИСТИКИ} \\ УСТАНОВОК ИОННОГО АЗОТИРОВАНИЯ ПРОМЫШЛЕННОГО ТИПА
}

\begin{abstract}
Аннотация. Приводится анализ энергетических характеристик промышленного оборудования - установок ионного азотирования, с различным исполнением вакуумных камер - с горячими и холодными стенками. Приведены результаты расчетов и экспериментальных исследований теплового баланса системы «садка - стенка камеры» при проведении процесса плазменного азотирования на установках промышленного типа. Рассмотрено влияние количества теплозащитных экранов в установках с холодными стенками на температуру внутреннего экрана и мощность тепловых потерь при разогреве садки деталей и ее изотермической выдержке. Показано существенное влияние геометрии разрядной камеры на величину мощности разряда, необходимой для разогрева садки и ее выдержки при определенной температуре - чем меньше геометрические размеры камеры (диаметр и высота), тем меньшая мощность тлеющего разряда нужна для обеспечения необходимой температуры садки. Показано, что при достаточном удалении садки деталей от стенок камеры, падение напряжения на остове тлеющего разряда может составлять десятки вольт, что приводит к снижению катодного падения потенциала и, соответственно, к увеличению электрической мощности, необходимой для поддержания требуемой температуры садки по сравнению с садкой, максимально приближенной к стенкам камеры. Это приводит к тому, что при более плотной загрузке требуется меньший удельный расход электроэнергии для обеспечения необходимой глубины азотированного слоя; при этом удельные энергозатраты при температуре садки $525-530{ }^{\circ} \mathrm{C}$ составляют величину $0,6-1,6$ кВт·ч/кг в зависимости от степени загрузки камеры. Показано, что при ионном азотировании давление рабочего газа должно быть таким, чтобы обеспечивалась аномальность тлеющего разряда, то есть свечением разряда должна быть охвачена вся площадь катода-садки.
\end{abstract}

Ключевые слова: ионно-плазменное азотирование, тепловой баланс, теплозащитный экран, мощность тлеющего разряда

Для цитирования. Босяков, М. Н. Энергетические и газодинамические характеристики установок ионного азотирования промышленного типа / М. Н. Босяков, А. А. Козлов // Вес. Нац. акад. навук Беларусі. Сер. фіз.-тэхн. навук. 2018. - Т. 63, № 3. - С. 342-350. https://doi.org/10.29235/1561-8358-2018-63-3-342-350

\author{
M. N. Bosyakov' ${ }^{1}$ A. A. Kozlov²

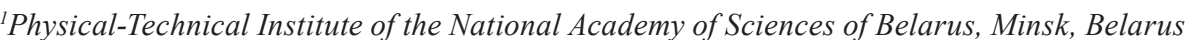 \\ ${ }^{2}$ Ministry of Industry of the Republic of Belarus, Minsk, Belarus
}

\title{
ENERGY AND GAS DYNAMIC CHARACTERISTICS OF IONIZATION NITROGEN INSTALLATIONS OF INDUSTRIAL TYPE
}

\begin{abstract}
The work provides the analysis of power characteristics of the industrial equipment - ionic nitriding applications - with different types of vacuum chambers - with hot and cold walls. The calculations results and experimental researches of thermal balance of the system "melt-wall chamber" at the process of plasma nitration for industrial applications are given. The work examines the influence of heat-shielding screens quantity in the applications with cold walls on the internal screen temperature and power of thermal losses at warming up parts melt and at its isothermal endurance. It has been displayed the significant influence of the discharge chamber geometry on the discharge power value which is necessary for the melt warming up and its exposition at the certain temperature - the less are geometrical sizes of the chamber (diameter and height), the smaller is the power of the smoldering discharge which is required for ensuring necessary melt temperature. It is shown that at sufficient melt parts distance from the chamber walls, the voltage drop on the skeleton of the smoldering discharge can be tens volts that causes the decrease of cathodic falling potential and, respectively, the increase of the electric power which is required to maintain necessary melt temperature in comparison to melt which is as close as possible to chamber walls. It leads to the fact that at more dense loading, smaller specific consumption of the electric power to ensure the necessary depth of the nitrated layer is required; at the same time the specific energy consumptions by the melt temperature of $525-530{ }^{\circ} \mathrm{C}$ are $0.6-1.6 \mathrm{~kW} \cdot \mathrm{h} / \mathrm{kg}$, depending on the loading extent of the chamber. It has been displayed that at ionic nitration, the value of working gas pressure must provide the deviance of the smoldering discharge i. e. the whole area the cathode -
\end{abstract}

(C) Босяков М. Н., Козлов А. А., 2018 
melt must be captured by the discharge luminescence.Keywords: ion-plasma nitriding, thermal balance, the heat-shielding screen, power of the smoldering discharge.

Keywords: ion-plasma nitration, thermal balance, the heat-shielding screen, power of the smoldering discharge

For citation. Bosyakov M. N., Kozlov A. A. Energy and gas dynamic characteristics of ionization nitrogen installations of industrial type. Vestsi Natsyyanal'nai akademii navuk Belarusi. Seryya fizika-technichnych navuk = Proceedings of the National Academy of Sciences of Belarus. Physical-technical series, 2018, vol. 63, no. 3, pp. 342-350 (in Russian). https://doi. org/10.29235/1561-8358-2018-63-3-342-350

Введение. Мировой опыт машиностроения последних 30-40 лет и собственный более чем 20-летний опыт авторов по разработке и внедрению технологии ионного азотирования в машиностроении показывает, что азотирование в плазме тлеющего разряда является наиболее современным и технологичным видом поверхностного упрочнения деталей машин (шестерен, зубчатых колес, вал-шестерен, пресс-форм, штампов и режущего инструмента). Этот метод позволяет получать изделия высокого качества с минимальными затратами на механическую и термическую обработку деталей.

Конструктивно установка ионного азотирования состоит из рабочей камеры, системы электропитания, газовакуумной системы и системы охлаждения. По конструкции рабочей камеры установки подразделяются на: колпаковые, колпаковые секционные, шахтные и дверные в зависимости от типа изделий, подлежащих упрочняющей обработке. На данный момент в мире используется два типа установок ионного азотирования: установки с пассивными горячими стенками производства фирмы Ionitech (Болгария) [1], Физико-технического института НАН Беларуси [2], Puls-Plasma Nitriding Units (Бразилия) [3] и установки с активными горячими стенками PVA Industrial Vacuum Systems GmbH (Plateg) (Германия) [4], ELTROPULS Heat-Treatment-Unit (Германия) [5], RÜBIG Heat Treatment (Австрия) [6]. В последнее время установки второго типа производит и фирма Ionitech [1]. В установках с горячими стенками нагрев садки деталей комбинированный - частично от стенок, а частично тлеющим разрядом. Цикл разогрева садки в таких установках начинается с повышения температуры стенки камеры, детали нагреваются от стенки до температуры $200-300{ }^{\circ} \mathrm{C}$ и более, а далее, при достижении в камере вакуума порядка 30-40 Па, зажигается разряд и происходит комбинированный нагрев. Скорость охлаждения определяется геометрией деталей и плотностью загрузки, и ее можно регулировать путем выбора метода охлаждения. Например, в камеру подается азот до определенного давления и включаются вентилятор, расположенный внутри камеры, а также вентиляторы снаружи камеры. В некоторых специальных случаях целесообразно проводить охлаждение в плазме, причем в данном случае существует возможность задавать нужный темп охлаждения, что можно осуществлять в установках с холодными и горячими стенками. Таким образом, в установках с горячими стенками для поддержания температуры садки на необходимом уровне допускается снижение мощности тлеющего разряда по сравнению с такой же камерой с холодными стенками. Однако детальное рассмотрение вопроса влияния уровня мощности тлеющего разряда на процесс ионного азотирования на установках промышленного типа практически отсутствует.

Целью данной работы является анализ энергетических и газодинамических (давление, расход газа, состав газовой смеси) характеристик установок промышленного типа с холодными и горячими стенками на основе анализа процесса теплообмена в вакуумной камере в системе «садка деталей - стенки рабочей камеры»

Параметры тлеющего разряда. Общим обязательным условием для всех типов установок с горячими и холодными стенками - является обеспечение аномальности тлеющего разряда при проведении процесса обработки независимо от давления в камере.

Аномальный тлеющий разряд - это форма тлеющего разряда, при которой свечением охвачена вся площадь катода-садки, а при повышении вкладываемой в разряд мощности вместе с ростом напряжения увеличивается и сила тока разряда.

Нормальный тлеющий разряд - это форма тлеющего разряда, при которой свечением охвачена только часть катода, а при увеличении вкладываемой мощности растет лишь разрядный ток, в то время как величина катодного падения потенциала $\left(U_{\text {кпा }}\right)$ остается неизменной. Это так называемое нормальное катодное падение потенциала, которое является первым параметром. 


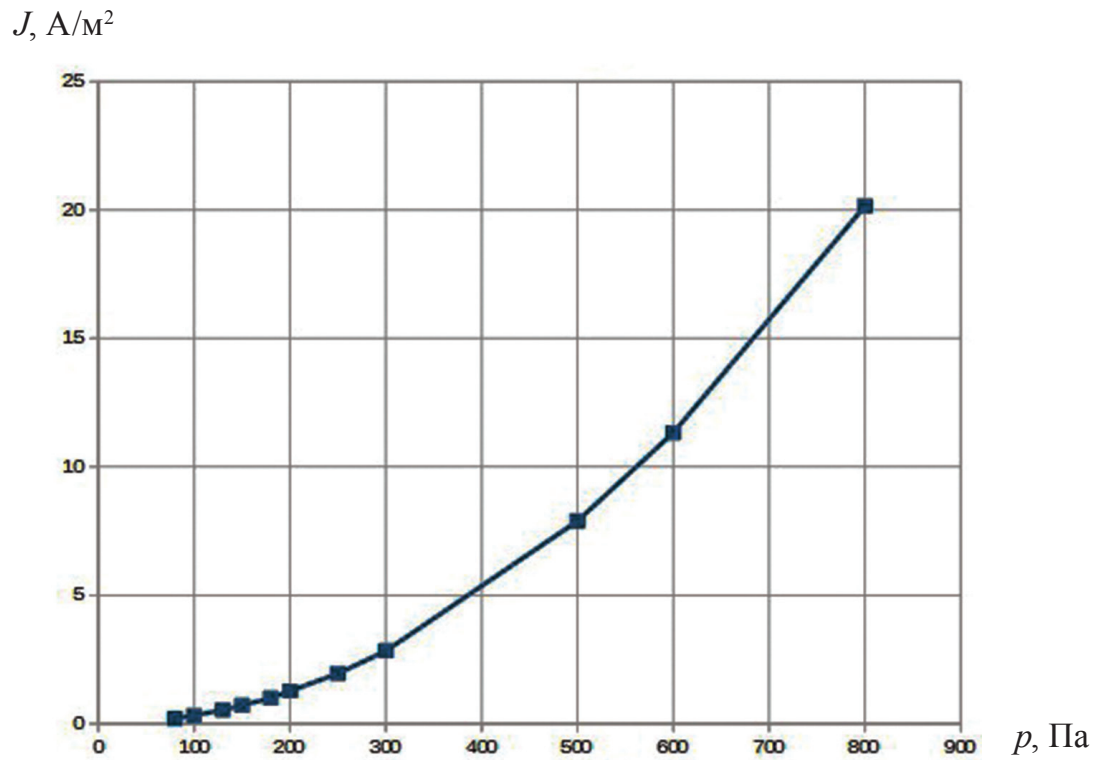

Рис. 1. Расчетное значение нормальной плотности тока тлеющего разряда в азоте в зависимости от давления

Fig. 1. Calculated value of current normal density of the smoldering discharge in nitrogen depending on pressure

Если катод изготовлен из сплава железа, то для азота значение $U_{\text {кпп }}$ составляет $215 \mathrm{~B}$, для водорода -250 В и для аргона - 165 В [7].

Вторым параметром, который позволяет разделять тлеющий разряд на нормальный и аномальный, является «нормальная плотность тока»- $J / p^{2}$, величина которой для катода из сплава железа при температуре $300 \mathrm{~K}$ для азота составляет $2,26 \cdot 10^{-4} \mathrm{~A} /\left(\mathrm{m}^{2} \cdot\right.$ Па $\left.{ }^{2}\right)$, для аргона $9,0 \cdot 10^{-5} \mathrm{~A} /\left(\mathrm{m}^{2} \cdot \mathrm{\Pi}^{2}\right)$ и для водорода $-4,1 \cdot 10^{-5} \mathrm{~A} /\left(\mathrm{m}^{2} \cdot \mathrm{Ma}^{2}\right)$ [7]. Следовательно, если реальное значение плотности тока в процессе обработки при конкретном давлении и температуре будет меньше, чем значение нормальной плотности тока для азота, который является основным компонентом рабочей смеси, то разряд может быть частично нормальным и частично аномальным, то есть на части садки разряд будет отсутствовать. Например, для температуры обработки 803 К расчетное значение нормальной плотности тока как функции давления представлено на рис. 1. Расчет проводился для приведенного давления с учетом нагрева газа в катодной области разряда [8]. Как следует из представленных данных, рост давления приводит к увеличению величины нормальной плотности тока в разряде и, к примеру, при обрабатываемой площади порядка $30 \mathrm{~m}^{2}$ при давлении 800 Па ток разряда должен быть не менее 600 А, чтобы разряд существовал в аномальном виде.

Таким образом, давление рабочего газа при ионном азотировании должно быть таким, чтобы обеспечивалась аномальность тлеющего разряда.

Взаимосвязь энергетических и технологических параметров процесса ионного азотирования. Рассмотрим более подробно взаимосвязь энергетических (напряжение и плотность тока) и технологических (режимных) (температура, давление и состав газовой среды) параметров процесса ионного азотирования. Параметры режима являются взаимосвязанными, поэтому энергетические характеристики не могут выбираться произвольно, так как для обеспечения, например, требуемой температуры для каждой конкретной садки и геометрии разрядной камеры устанавливаются некоторые комбинации энергетических показателей разряда - силы тока и напряжения разряда. При назначении режима обработки руководствуются прежде всего требованиями конструкторской документации по параметрам азотированного слоя - глубине и поверхностной твердости. Следует отметить, что на некоторые детали задается также дополнительно требование - наличие на заданной глубине определенной твердости, что является, по сути, требованием к профилю твердости в азотированном слое. Температура изотермической выдержки назначается из расчета обеспечения необходимой поверхностной твердости детали (которая тем выше, чем ниже температура [9]) и исходя из соображений экономического характера - обеспечения необ- 
ходимой производительности оборудования. Следующим этапом является выбор рабочего давления и состава газовой смеси, а также конкретных расходов каждого компонента этой смеси, в особенности азота.

Рассмотрение взаимосвязи параметров процесса обработки - энергетических и режимных целесообразно провести на основе анализа теплообмена в вакуумной камере в системе «садка деталей - стенки рабочей камеры», поскольку в стационарном режиме (при изотермической выдержке) именно мощность тепловых потерь садки будет определять энергетику процесса независимо от того, горячая стенка камеры или холодная. Согласно [10], под камерой с холодными стенками подразумевается вакуумная камера с рубашкой водяного охлаждения, хотя камеры установок ряда производителей оборудования имеют, помимо рубашки охлаждения, также либо экранную теплоизоляцию, либо комбинированную с использованием экранов и волокнистых теплоизоляционных материалов на основании камеры и ее потолке (крышке) [1].

Независимо от того, как выполнена вакуумная камера - с горячими или с холодными стенками, - мощность тлеющего разряда на стадии выдержки будет определяться тепловыми потерями камеры, то есть теплообменом между садкой (обрабатываемыми деталями) и стенкой камеры.

При этом предполагается, что в тлеющем разряде мощность, выделяющаяся на катоде, которым является обрабатываемое изделие, и приводящая к его нагреву, определяется в основном энергией, приносимой потоком положительных ионов и «быстрых» молекул и атомов, что образуются при перезарядке в области катодного падения потенциала. Согласно [7], доля мощности, идущая на разогрев катода (садки), составляет от 0,8 до 0,9 от общей мощности разряда

$$
P_{\text {эл }}=U I k,
$$

где $U$ - напряжение, $I$ - сила тока разряда, $k$ - коэффициент заполнения (для пульсирующего разряда).

Мощность, затрачиваемая на нагрев катода, определяется выражением [7]

$$
W_{\text {нагр }}=j U_{\text {кпп }} \text {, }
$$

где $j$-плотность тока, а $U_{\text {кпп }}$ - катодное падение потенциала. Как правило, величина $U_{\text {кпп }}$ составляет значение от 80 до 90 \% приложенного к электродам напряжения в зависимости от давления и состава плазмообразующего газа [7], и для оценки можно использовать среднее значение 0,85 . Следует отметить, что $U_{\text {кпп }}$ всегда меньше приложенного к катоду напряжения и зависит от расстояния «деталь - стенка камеры» и степени аномальности разряда, но должно превышать величину нормального катодного падения потенциала [7]. Приложенное к катоду напряжение $U_{\text {разр }}$ (напряжение горения разряда) равно сумме падений напряжения на отдельных участках разряда - $U_{\text {кпп, }} U_{\text {a }}$ (анодное падение потенциала, которое, как правило, принимают на уровне потенциала ионизации газа [11]) и $U_{\text {ст }}$ (падение напряжения на положительном столбе разряда). Поскольку положительный столб в разряде при реальных режимах обработки практически отсутствует, то, согласно [11], эта зона разряда классифицируется как остов, причем падение напряжения на нем составляет единицы вольт на 10 мм длины остова [11]. Это значит, что при достаточном удалении садки деталей от стенок камеры падение напряжения на остове может составлять десятки вольт и катодное падение потенциала может быть меньше, чем 80 \%, что приведет к увеличению электрической мощности, необходимой для поддержания требуемой температуры садки по сравнению с садкой, максимально приближенной к стенкам камеры. Оптимальным является расстояние между стенкой и садкой на уровне 75-80 мм.

В случае камеры с горячими стенками дополнительным источником тепла при нагреве и выдержке садки являются нагреватели на стенке, поэтому в таких камерах доля энерговклада от разряда будет меньше, чем в установках с холодными стенками. В данном случае нужно выбирать определенный баланс между температурой стенки и мощностью тлеющего разряда, который будет обеспечивать необходимую химическую активность разряда.

При разогреве садки энергия тлеющего разряда расходуется на повышение теплосодержания деталей в садке и оснастки (если таковая используется), а также элементов камеры (экранов и стенки камеры). 
При этом для установок с холодными стенками температура стенки камеры предполагается постоянной, так как она является водоохлаждаемой и не должна быть выше $45{ }^{\circ} \mathrm{C}$ (ГОСТ 12.2.007.9-93. Безопасность электротермического оборудования. Ч. 1. Общие требования), а излучающей поверхностью считается поверхность, огибающая нагреваемую садку. Следует заметить, что при обработке, например, шестерен с зубом на внутреннем венце азотируемая площадь может быть существенно больше площади, участвующей в теплообмене со стенками камеры. При разогреве садки необходимо также учитывать увеличение теплоемкости стали с ростом температуры. Для установок с горячими стенками температура внутренней стенки поддерживается нагревателями, однако часть тепла на стенку поступает также и от садки вследствие излучения. Если задан темп разогрева, то уравнение баланса энергии при разогреве садки можно представить в виде

$$
\mathrm{cm} \frac{d T}{d t}=P_{\text {нагр }}-P_{\text {охл }},
$$

где $c$ - теплоемкость стали (для углеродистой стали в интервале температур $50-550{ }^{\circ} \mathrm{C}$, среднее значение теплоемкости $c=0,162 \mathrm{BT} \cdot{ }^{-} \cdot \kappa \Gamma^{-1 .}{ }^{\circ} \mathrm{C}^{-1}$, для оценочных расчетов можно использовать среднее значение теплоемкости стали, однако при более точных расчетах необходимо учитывать температурную зависимость теплоемкости); $m$ - масса садки, кг; $\frac{d T}{d t}-$ темп разогрева, ${ }^{\circ} \mathrm{C} / \mathrm{ч} ; P_{\text {нагр }}$ и $P_{\text {охл }}$ - соответственно мощность, идущая на нагрев садки и теряемая ею при охлаждении вследствие теплообмена со стенками, Вт. Если считать, что охлаждение садки происходит вследствие лучистого теплообмена со стенками камеры, то тогда $P_{\text {охл }}=Q_{(\mathrm{c}, \mathrm{c \tau}) 3}$ и поток излучения $Q_{(\mathrm{c}, \mathrm{c \tau}) 3}$ от садки с излучающей площадью $F_{\mathrm{c}}$ и температурой $T_{\mathrm{c}}$ к стенке, имеющей температуру $T_{\text {ст }}$, при наличии теплозащитных экранов будет выглядеть следующим образом [12]:

$$
Q_{(\mathrm{c}, \mathrm{cт}) \ni}=C_{0} A_{(\mathrm{c}, \mathrm{cr})} F_{\mathrm{c}}\left(\left[\frac{T_{\mathrm{c}}}{100}\right]-\left[\frac{T_{\mathrm{c \tau}}}{100}\right]\right),
$$

где $A_{(\mathrm{c}, \mathrm{c}) э}-$ приведенный коэффициент поглощения рассматриваемой излучающей системы, имеющий различные значения для случая цилиндрических экранов и плоских экранов; $C_{0}-$ константа излучения черного тела, $C_{0}=5,67 \mathrm{BT}^{*} \mathrm{M}^{2} \cdot \mathrm{K}^{4}$.

Как следует из выражения (2), поток излучения зависит от разности температур излучающего тела (в нашем случае - садки) и стенки камеры (либо экрана), площади излучающей поверхности и приведенного коэффициента поглощения $A_{(\mathrm{c}, \mathrm{c \tau )})}$, и по мере увеличения температуры садки поток тепла на стенку возрастает, причем его величина пропорциональна излучающей площа-

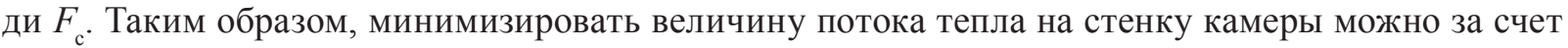
применения эффективной системы теплоизоляции, то есть использования большого количества

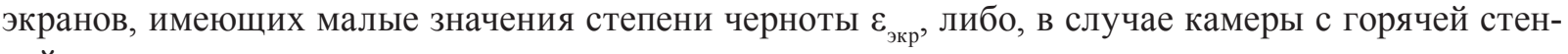
кой, поддерживать температуру стенки на некотором уровне.

Таким образом, из (1) и (2) выражение для определения необходимой электрической мощности разряда (при условии, что 85 \% расходуется на нагрев садки) будет иметь вид при разогреве садки

$$
P_{\text {эл }}=1,18\left(\mathrm{~cm} \frac{d T}{d t}+Q_{(\mathrm{c}, \mathrm{cт}) э}\right)
$$

и при выдержке, когда $T=$ const,

$$
P_{\text {эл }}=1,18 Q_{(\mathrm{c}, \mathrm{c} \mathrm{c})} \text {. }
$$

Если камера имеет горячую стенку, то величина $A_{(\text {с,ст) }}$ определяется следующим образом [13]:

$$
A_{(\mathrm{c}, \mathrm{cr})}=1 /\left(\varepsilon_{\mathrm{c}}^{-1}+\left(\varepsilon_{\mathrm{cT}}^{-1}-1\right)\left(F_{\mathrm{c}} / F_{\mathrm{cr}}\right)\right),
$$

где $\varepsilon_{\mathrm{c}}$ и $\varepsilon_{\text {ст }}$ - соответственно степень черноты садки и стенки, $F_{\text {с }}$ и $F_{\text {ст }}$ - площади излучающей поверхности садки и стенки соответственно. 
В случае камеры с холодными стенками для системы цилиндрических экранов выражение для $A_{(\mathrm{c}, \mathrm{cr})}$ имеет вид

$$
\left.A_{(\mathrm{c}, \mathrm{cr})}=1 /\left(\varepsilon_{12}^{-1}+\sum\left(F_{\mathrm{c}} / F_{\ni i}\right)\left(2 / \varepsilon_{3 i}^{-1}-1\right)+F_{\mathrm{c}} / F_{\mathrm{cT}}\right)\right),
$$

где $\varepsilon_{12}$ - приведенная степень черноты системы «садка - стенка», определяемая по формуле (5), $F_{э i}$ и $\varepsilon_{э i}$ - площадь и степень черноты соответствующего $i$-го экрана.

Для системы плоских экранов (основание и верх камеры) выражение для $A_{(\mathrm{c}, \mathrm{cr})}$ следующее:

$$
A_{(\mathrm{c}, \mathrm{cT})}=1 /\left(\varepsilon_{\mathrm{c}}^{-1}+2 \sum \varepsilon_{э i}^{-1}+\varepsilon_{\mathrm{cт}}^{-1}-(n+1)\right),
$$

где $n$ - количество экранов.

На основании данных из $[14,15]$ в расчетах принимались следующие значения величин $\varepsilon$ : $\varepsilon_{\mathrm{c}}=0,4 ; \varepsilon_{\text {ст }}=0,6 ; \varepsilon_{31}=0,6 ; \varepsilon_{32}=0,56$ и $\varepsilon_{33}=0,45$.

Теплообмен в камере. Вакуумные камеры установок ионного азотирования имеют различную геометрию: большой диаметр и небольшую высоту либо наоборот - малый диаметр и большую высоту (или глубину). Следовательно, у камер с большим диаметром основные потери тепла будут через верх и низ садки, а у камер с превалированием длины над диаметром - через боковую стенку. Как следует из приведенных формул для расчета величины $A_{(\mathrm{c}, \mathrm{cr})}$, существенное влияние на величину мощности разряда, необходимой для разогрева садки и ее выдержки при определенной температуре, оказывает геометрия разрядной камеры - чем меньше ее геометрические размеры (диаметр и высота), тем меньшая мощность необходима для обеспечения оптимальной температуры садки. Таким образом, по мере увеличения геометрических размеров камеры - диаметра и высоты (глубины), увеличивается и мощность разряда, которую необходимо прикладывать при проведении процесса обработки при одной и той же температуре выдержки. Эти факторы необходимо учитывать при проектировании установок ионного азотирования.

Расчет и экспериментальные исследования теплообмена в камере проводились применительно к установке ионного азотирования промышленного типа, с рабочей камерой колпакового типа с размерами рабочего пространства: диаметр - 950 мм, высота загрузки - 1250 мм. Камера имеет водоохлаждаемую рубашку, внутри камеры - экранная теплоизоляция (три экрана из листовой стали). Мощность тлеющего разряда на установке - до 50 кВт, при этом максимальное напряжение горения разряда - не более $700 \mathrm{~B}$, сила тока - 95 А. Загрузка камеры - до 1000 кг.

Расчеты, проведенные для различного количества теплозащитных экранов (одного, двух и трех), показали [16], что наличие в камере трех экранов снижает расход электроэнергии на стадии разогрева на 30-90 \% по сравнению с одним или двумя экранами.

Из соотношения (6) были получены расчетные значения температуры внутреннего, обращенного к садке экрана в зависимости от температуры садки при ее излучающей площади $F_{\mathrm{c}}=4,2 \mathrm{~m}^{2}$, а также от величины излучающей площади садки $F_{\mathrm{c}}$ при постоянной температуре садки $T=530{ }^{\circ} \mathrm{C}$. Эти данные и экспериментальные результаты измерения температуры экрана представлены на рис. $2-4$.

На рис. 4 показано изменение температуры внутреннего экрана, измеренной хромель-алюмелевой термопарой, в зависимости от температуры садки.

Высокая температура внутреннего экрана обеспечивает однородность температурного поля в садке и низкий расход электроэнергии. Так, при обработке вышеупомянутой садки на стадии выдержки затрачиваемая на поддержание разряда удельная мощность составляет всего 35-36 Вт/кг, а для садки деталей, представленной на рис. $5,-25-26$ Вт/кг. В установках ионного азотирования, имеющих один или два экрана, затраты мощности на поддержание разряда выше, так как теплообмен со стенками камеры интенсивнее и, соответственно, температура внутреннего экрана ниже, что приводит к существенному градиенту температуры садки в радиальном направлении.

При обработке садок деталей с различной степенью загрузки рабочей камеры было экспериментально установлено, что чем полнее загружена камера, тем меньше удельный расход электроэнергии для обеспечения необходимой глубины азотированного слоя, при этом удельные энергозатраты при температуре садки $525-530^{\circ} \mathrm{C}$ составляют величину $0,6-1,6$ кВт·ч/кг в зависимости от загрузки камеры. Кроме того, при расчете теплообмена необходимо учитывать тот 


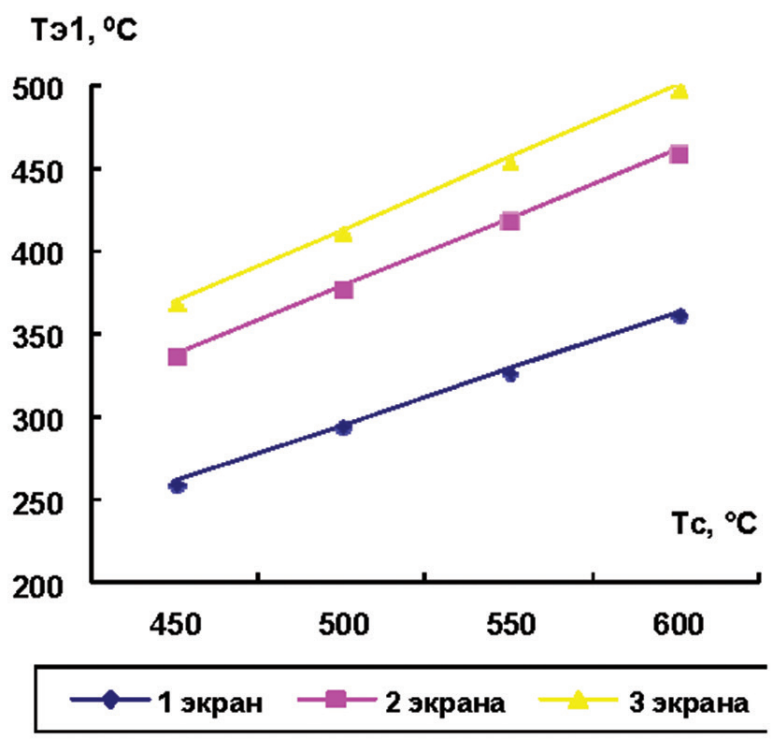

$\mathbf{T} 31 .{ }^{\circ} \mathrm{C}$

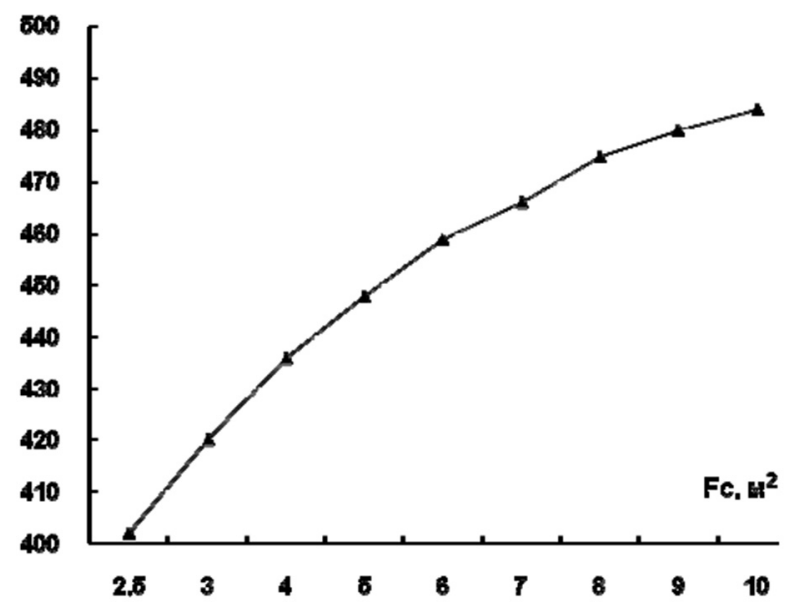

$\Delta \mathrm{T}=\mathrm{T}_{\text {садки }}-\mathrm{T}_{\text {экрана }}$

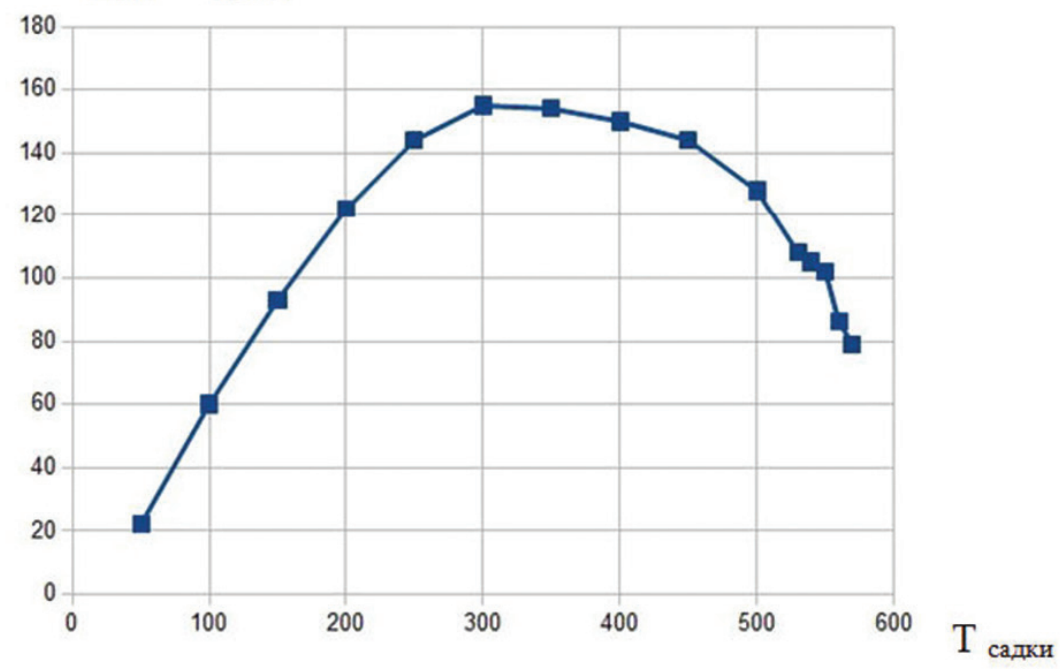

Рис. 4. Разность температур между садкой и внутренним экраном в зависимости от температуры садки

Fig. 4. The temperatures difference between melt and the internal screen depending on melt temperature
Рис. 2. Температура внутреннего экрана в зависимости от температуры садки для разного количества теплозащитных экранов в камере

Fig. 2. Internal screen temperature depending on melt temperature for the different number of heat-shielding screens in the chamber
Рис. 3. Температура внутреннего экрана в зависимости от излучающей площади садки $F_{\text {c }}$

Fig. 3. Internal screen temperature depending on the radiating melt area $F_{\mathrm{c}}$ 
Рис. 5. Садка шестерен с внутренним зацеплением с общей азотируемой площадью $24 \mathrm{~m}^{2}$

Fig. 5. Gear wheels melt with internal gearing with the total nitrated area $24 \mathrm{~m}^{2}$

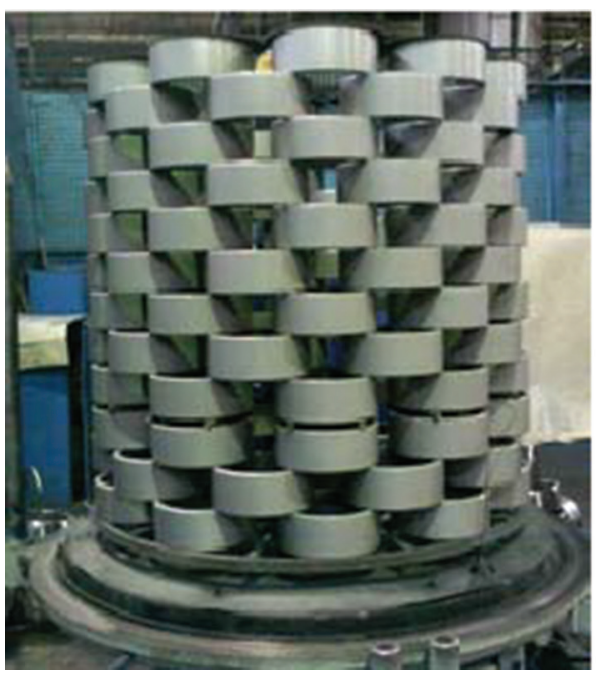

фактор, что в реальной садке имеет место облучение стенки камеры от разных элементов садки. Например, на представленной на рис. 5 садке шестерен в количестве 120 штук с внутренним зубом около 70 \% наружной площади каждой шестерни участвует в теплообмене со стенкой камеры - и эта суммарная площадь превышает излучающую площадь садки, принимаемую как боковая поверхность цилиндра с диаметром катода и высотой загрузки деталей.

Как показал анализ режимов азотирования садок на промышленном оборудовании, диапазон изменения параметра $J / p^{2}$ достаточно узок и величина катодного падения потенциала лишь на 5-10 \% превышает значение нормального катодного падения для азота. Следовательно, определяющую роль в формировании химической активности разряда на стадии изотермической выдержки, наряду с содержанием азота в смеси с другими газами, должна играть плотность тока разряда, которая зависит от вольт-амперной характеристики разряда (мощности тепловых потерь) и давления в камере.

Заключение. Проведенные расчеты теплообмена в системе «садка деталей - стенки рабочей камеры» показали зависимость мощности тлеющего разряда от теплообмена, наличия теплозащитных экранов и геометрии камеры.

При выборе рабочего давления на стадии изотермической выдержки необходимо учитывать особенности аномального тлеющего разряда, в результате давление разряда должно быть таким, чтобы гарантировать превышение реальной плотности тока над плотностью тока нормально тлеющего разряда.

\section{Список использованных источников}

1. IonitechLtd. Products [Electronic resource]. - Mode of access: http://www.ionitech.com/products/coldwall-equipment. html - Date of access: 01.06.2018.

2. Упрочняющая ионная химико-термическая обработка [Электронный ресурс] // Физико-технический институт НАН Беларуси. - Режим доступа: http://phti.by/product/Hardening-ion-chemical-heat-treatment. - Дата доступа: 01.06.2018.

3. Plasma Nitriding Equipment for South America [Electronic resource]. - Mode of access: https://www.secowarwick. com/en/news/plasma-nitriding-equipment-for-south-america/ - Date of access: 27.04.2014.

4. PVA Industrial Vacuum Systems GmbH. PulsPlasma ${ }^{\circledR}$ Nitriding. Oxidation [Electronic resource]. - Mode of access: http://www.plateg.com/willkommen-eng.html - Date of access: 01.06.2018.

5. Nitriding Plants [Electronic resource] // Eltropuls. - Mode of access: http://www.eltropuls.de/en/products/plant-engineering/nitriding - Date of access: 15.05.2018

6. Nitriding and Coating Systems [Electronic resource] // RÜBIG Industrial Furnaces. - Mode of access: https://www. rubig.com/fileadmin/user_upload/AT/Downloads/AT_Folder_Anlagentechnik_A4_EN_20170321_Einzelseiten.pdf - Date of access: 25.05.2017.

7. Энгель, А. Физика и техника электрического разряда в газах / А. Энгель, М. Штенбек. - М.: ОНТИ-НКТП, 1936. - Т. 2. -384 c.

8. Параметры области катодного падения потенциала самостоятельного нормального тлеющего разряда в гелии при атмосферном давлении / В. И. Архипенко [и др.] // Физика плазмы. - 2002. - Т. 28, № 10. - С. 858-865. https://doi. org/10.1134/1.1513839 
9. Чаттерджи-Фишер, Р. Азотирование и карбонитрирование / Р. Чаттерджи-Фишер, Ф. Эйзелл. - М.: Металлургия, 1990. - 280 c.

10. Pye, D. Practical Nitriding and Ferritic Nitrocarburizing / D. Pye. - ASM International Park, 2003. -256 p.

11. Капцов, Н. А. Электрические явления в газах и вакууме / Н. А. Капцов. - М.; Л.: ОГИЗ, 1947. - 810 с.

12. Исаченко, В. П. Теплопередача / В. П. Исаченко, В. А. Осипова, А. В. Сукомел. - М.: Энергоиздат, 1981. -415 с.

13. Теория, конструкции и расчеты металлургических печей: [в 2 т.] / под ред. В. А. Кривандина. - М.: Металлургия, 1986. - Т. 2: Расчеты металлургических печей / Б. С. Мастрюков. - 376 с.

14. Свенчанский, А. Д. Электрические промышленные печи: в 2 ч. / А. Д. Свенчанский. - М.: Энергия, 1975. Ч. 1: Электрические печи сопротивления. -384 с.

15. Блох, А. Г. Теплообмен излучением: справочник / А. Г. Блох, Ю. А. Журавлев, Л. Н. Рыжков. - М.: Энергоатомиздат, 1991. - $432 \mathrm{c}$.

16. Босяков, М. Н. Энергетические параметры процессов ионного азотирования на промышленном оборудовании / М. Н. Босяков, А. А. Козлов // Доклады БГУИР. - 2013. - № 3(73). - С. 76-82.

\section{References}

1. Ionitech Ltd. Products. Available at: http://www.ionitech.com/products/coldwall-equipment.html (Accessed 1 June 2018).

2. Strengthening ionic chemical-thermal treatment. The Physical-Technical Institute of the National Academy of Sciences of Belarus. Available at: http://phti.by/product/Hardening-ion-chemical-heat-treatment (Accessed 1 June 2018) (in Russian).

3. Plasma Nitriding Equipment for South America. Available at: https:/www.secowarwick.com/en/news/plasma-nitriding-equipment-for-south-america/ (Accessed 27 April 2014).

4. PVA Industrial Vacuum Systems GmbH. PulsPlasma ${ }^{\circledR}$ Nitriding. Oxidation. Available at: http://www.plateg.com/willkommen-eng.html (Accessed 1 June 2018).

5. Nitriding Plants. Eltropuls. Available at: http://www.eltropuls.de/en/products/plant-engineering/nitriding (Accessed 15 May 2018).

6. Nitriding and Coating Systems. RÜBIG Industrial Furnaces. Available at: https://www.rubig.com/fileadmin/user_upload/AT/Downloads/AT_Folder_Anlagentechnik_A4_EN_20170321_Einzelseiten.pdf (Accessed 25 May 2017).

7. Engel' A., Shtenbek M. Physics and Technology of Electric Discharge in Gases. Volume 2. Moscow, ONTI-NKTP Publ., 1936. 384 p. (in Russian).

8. Arkhipenko V. I., Zgirovskii S. M., Kirillov A. A., Simonchik L. V. Cathode Fall Parameters of a Self-Sustained Normal Glow Discharge in Atmospheric-Pressure Helium. Plasma Physics Reports, 2002, vol. 28, iss. 10, pp. 858-865. https:// doi.org/10.1134/1.1513839

9. Chatterdzhi-Fisher R., Eizell F. Nitriding and carbonitriding. Moscow, Metallurgiya Publ., 1990. 280 p. (in Russian).

10. Pye D. Practical Nitriding and Ferritic Nitrocarburizing. ASM International Park, 2003. 256 p.

11. Kaptsov N. A. Electrical phenomena in gases in vacuum. Moscow, Leningrad, Association of state book and magazine Publishing Houses, 1947. 810 p. (in Russian).

12. Isachenko V. P., Osipova V. A., Sukomel A. V. Heat Transfer. Moscow, Energoizdat Publ., 1981. 415 p. (in Russian).

13. Mastryukov B. S. Theory, constructions and metallurgical furnace calculations. Vol. 2: Metallurgical furnace calculations. Moscow, Metallurgiya Publ., 1986. 376 p. (in Russian).

14. Svenchanskii A. D. Electric industrial furnaces. Part 1: Electrical furnace of resistance. Moscow, Energiya Publ., 1975. 384 p. (in Russian).

15. Blokh A. G., Zhuravlev Yu. A., Ryzhkov L. N. Heat exchange by radiation. Moscow, Energoatomizdat Publ., 1991. 432 p. (in Russian).

16. Bosyakov M. N., Kozlov A. A. The energy parameters of the processes of ion nitriding on industrial equipment. Doklady BGUIR [Reports BSUIR], 2013, no. 3 (73), pp. 76-82 (in Russian).

\section{Информация об авторах}

Босяков Михаил Никифорович - кандидат физикоматематических наук, ведущий научный сотрудник лаборатории электрофизики, Физико-технический институт Национальной академии наук Беларуси (ул. Купревича, 10, 220141, Минск, Республика Беларусь). E-mail: plasma.by.metal@gmail.com

Козлов Алексей Александрович - начальник научнотехнического управления, Министерство промышленности Республики Беларусь (пр. Партизанский, 2-4, 220033 , Минск, Республика Беларусь). E-mail: alex-ett@tut.by

\section{Information about the authors}

Michail N. Bosyakov - Ph. D. (Physics and Mathematics), Leading Researcher of the Laboratory of Electrophysics, Physical-Technical Institute of the National Academy of Sciences of Belarus (10, Kuprevich Str., 220141, Minsk, Republic of Belarus).E-mail: plasma.by.metal@gmail.com

Alexej A. Kozlov - Chief of Scientific and Technical Department, Ministry of Industry of the Republic of Belarus (2, building 4, Partizansky Ave., 220033, Minsk, Republic of Belarus).E-mail: alex-ett@tut.by 